\title{
BEARING ESTIMATION USING DOUBLE FREQUENCY REASSIGNMENT FOR A LINEAR PASSIVE ARRAY
}

\author{
Krzysztof Czarnecki \\ Wojciech Leśniak \\ Gdansk University of Technology
}

\begin{abstract}
The paper demonstrates the use of frequency reassignment for bearing estimation. For this task, signals derived from a linear equispaced passive array are used. The presented method makes use of Fourier transformation based spatial spectrum estimation. It is further developed through the application of two-dimensional reassignment, which leads to obtaining highly concentrated energy distributions in the joint frequency-angle domain and sharp graphical imaging. The introduced method can be used for analysing, a priori, unknown signals of broadband, nonstationary, and/or multicomponent type. For such signals, the direction of arrival is obtained based upon the marginal energy distribution in the angle domain, through searching for arguments of its maxima. In the paper, bearing estimation of three popular types of sonar pulses, including linear and hyperbolic frequency modulated pulses, as well as no frequency modulation at all, is considered. The results of numerical experiments performed in the presence of additive white Gaussian noise are presented and compared to conventional digital sum-delay beamforming performed in the time domain. The root-mean-square error and the peak-to-average power ratio, also known as the crest factor, are introduced in order to estimate, respectively, the accuracy of the methods and the sharpness of the obtained energy distributions in the angle domain.
\end{abstract}

Keywords: Direction of arrival, DOA, instantaneous frequency, short-time Fourier transform, STFT, time-frequency reassignment, intercept and surveillance sonar, crest factor

\section{INTRODUCTION}

Bearing estimation is an important issue in sonar and radar techniques [9], [21]. In underwater acoustic systems, the direction of arrival (DOA) of the signal can be estimated, among others, through the use of its spatial spectrum [4], [10], [15]. The considerations presented in the paper exploit and expand this approach by introducing two-dimensional reassignment in a joint frequency-angle domain, which is a novel modification, compared to classical time-frequency reassignment [11]. The main aim of this research is to present the reassignment as a usable method for DOA estimation and to assess its utility in this field. We propose the use of this method especially in order to analyse nonstationary and multicomponent signals whose parameters are $a$ priori unknown, as well as by allowing comprehensive graphical presentation. These issues are particularly important in the considered passive sonar system. Other critical requirements for digital real-time systems include predictable and short durations of their response, as well as their high performance and stability. These requirements can be achieved by using Fast Fourier Transformation (FFT) which is the implementation basis of the proposed method [22].

The reassignment method, initially referred to as "the modified moving window method", was introduced by Kodera et al. in 1976 [11]. In general, this approach is helpful in obtaining highly concentrated energy distributions in the joint time-frequency domain and can be effectively used for 
analysing multicomponent and non-stationary signals with components sparsely distributed over the time-frequency plane. Numerous authors have proposed contributions to the aforementioned approach [1], [5], [7], to name but a few. The time-frequency reassignment is accomplished through energy relocation, which employs local group delay (LGD) and channelized instantaneous frequency (CIF). In the paper, we consider the reassignment in the frequency-angle domain, or equivalently, in the frequency-bearing domain. Therefore, we still use the CIF, but the LGD will be knowingly overlooked. Meanwhile, DOA can be obtained by the ratio of CIF and local spatial frequency (LSF). Both, CIF and LSF, may be estimated by differentiating the phase of the extended short-time Fourier transform (STFT). We propose extending the classical STFT to four dimensions: time, frequency, propagation delay, and spatial frequency. This allows us to obtain CIF and LSF by processing a single transform. The proposed method is novel, but there are other contributions to bearing estimation in this particular context, which have also been reported in [3] and [6].

The introduced method and the used linear passive array are presented as elements of a surveillance or intercept sonar system. All the relevant situations and analysed signals are related, in some way, to this area. Nonetheless, the resultant conclusions are universal and may also be applicable to other areas of bearing estimation, where the received signals stored by a linear array consist of a number of omnidirectional sensors. The method is also referred to time-frequency reassignment as its extension to many dimensions.

The paper is organized as follows. In Section II, a general scheme of data acquisition and forming the received signal in a system with linear passive array is presented. In Section III, the concept of a novel method of bearing estimation is introduced. Section IV contains descriptions of its discrete implementations. Finally, in Section V, the results of numerical experiments and analyses of popular types of sonar sounding pulses with and without frequency modulation are presented. The accuracy of the method and the sharpness of the final imaging in the presence of noise are also evaluated in this section.

\section{LINEAR PASSIVE ARRAY}

From a military point of view, water depth can be explored by sonars in search of potentially dangerous objects such as submarines, torpedoes, and mines. However, active sonar systems are usually very reluctantly used by the crews of Navy ships during military operations. When functioning, the active sonar easily gives away its location. Furthermore, a sounding signal must have a sufficient amount of energy to allow back and forth propagation between the sonar and the target, and must take into account any losses due to reflection and backscatters. This is the reason why the distance over which a sounding sonar can detect targets is significantly smaller than the distance from which this sonar can be detected by other passive sonar systems. Conversely, passive systems do not possess this disadvantage and are important equipment used by Navy vessels, especially out on the high seas. However, it should be added that the passive systems are helpless when facing objects which do not emit any acoustic signals, mines for instance. Another source of restrictions in the use of active sonar systems is their adverse effects on underwater fauna [19]. This is particularly true of systems emitting high-power acoustic waves into the natural environment. Therefore, development in this area is crucial and is largely driven by practical considerations [14].

Let us consider a hypothetical passive sonar system with a linear array of omnidirectional hydrophones [12], [20]. This array consists of $M$ hydrophones uniformly distributed along a straight line, at a distance from each other equal to $d_{\Delta}$. In this way, each received acoustic signal can be uniformly sampled in both space and time. This means that the Nyquist-Shannon sampling theorem should be met in all these dimensions. In the time domain, the maximal frequency $f_{\max }$ of the signal component in the Fourier spectrum is only limited by the sampling rate $r_{s}$, in accordance with $f_{\max }<r_{s} / 2$. Respectively, in space, the maximal frequency depends on the distance between the neighbouring hydrophones $d_{\Delta}$, the propagation velocity of the acoustic wave in water $v_{w}$, and the direction of arrival $\phi_{u}$ of the signal $u(t)$. In the situation under consideration, signal parameters, especially the DOA, are, a priori, unknown. Therefore, $d_{\Delta}$ should be set taking into account the reception of a signal propagated along the array, due to the fact that this direction is most demanding as regards sampling. Consequently, the greatest satisfactory distance between the neighbouring hydrophones is equal to:

$$
d_{\Delta}=v_{w} /\left(2 f_{\max }\right)
$$

where $f_{\max }$ is the maximal expected frequency of a considerable component in the Fourier spectrum of the incoming signal. In general, the resolution of bearing estimation in the classical approach is greater if the array is longer. Unfortunately, increasing the number of hydrophones can, simultaneously, increase significantly the overall cost of the entire system. Consequently, $d_{\Delta}$ is usually assumed as to be as large as possible, taking into account the fact that it is a relatively inexpensive means to elongate the array, as the total array length is equal to $M d_{\Delta}$ as well as the whole delay along the longitudinal section of the array being

$$
\tau_{\text {arr }}=M d_{\Delta} / v_{w}
$$

The surveillance sonar under consideration is usually used for receiving signals whose sources are located at a significant distance from the array. This distance should be far greater than the wavelength of the received signal - the array is located in the far acoustic field. The arrival signals, including useful, disturbing, and noise contributions from various sources and directions, are summed and saved into the buffers. The scheme of input sample acquisition is 
presented in Figure 1. The saved samples can be treated as the received signal $u(t, \tau)$ of two variables, dependent on time $t$ and delay along the longitudinal section of the array $\tau$ as presented in Figure 2.

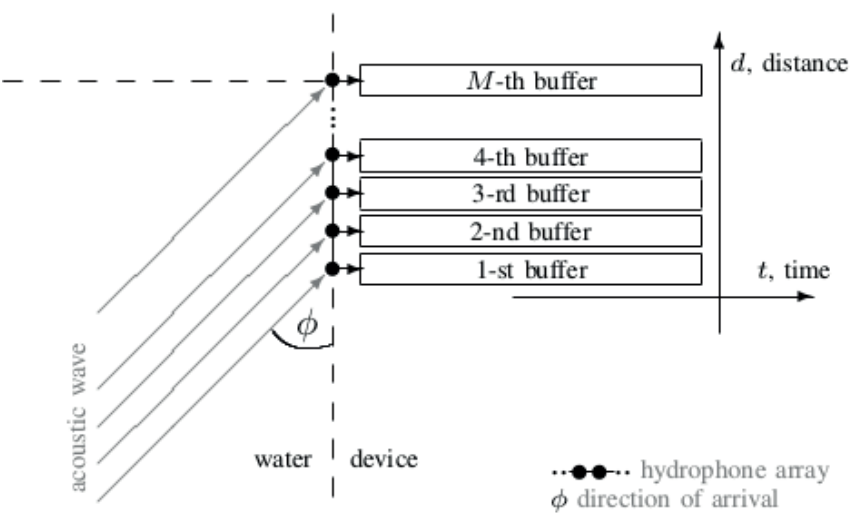

Fig. 1. Schema of data acquisition in a passive array.

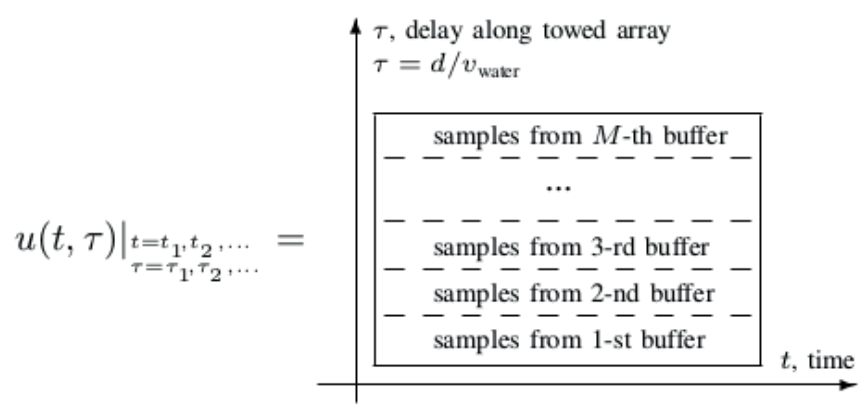

Fig. 2. Schema of received discrete signal forming.

\section{DOUBLE FREQUENCY REASSIGNMENT}

Let us consider the following complex-valued fourdimensional variant of the short-time Fourier transform of the function $u(t, \tau)$ of two variables:

$$
\begin{aligned}
& U_{h}(t, \tau, \omega, \varpi)=A_{h}(t, \tau, \omega, \varpi) \exp \left(j \varphi_{h}(t, \tau, \omega, \varpi)\right) \\
& \iint u(\varsigma, \xi) \bar{h}(\varsigma-t, \xi-\tau) \exp (-j(\varsigma \omega+\xi \varpi)) d \xi d \varsigma .
\end{aligned}
$$

It turns out that $U_{h}(t, \tau, \omega, \omega)$ is a function of the following four variables: time $t$, geometrical location along the array expressed by propagation delay $\tau$, angular frequency $\omega$, and spatial angular frequency $\omega . A_{h}(t, \tau, \omega, \omega)$ is the amplitude and $\varphi_{h}(t, \tau, \omega, \omega)$ is the appropriate phase of the Fourier transform. $h(t, \tau)$ with a short line over it expresses the conjugated function of the analysing tapering window [6].

The Blackman-Harris (BH) window, which is a function of two variables: time and propagation delay, is used here as the analysing window. It is limited (finite) both in time and in delay. In the situation in question, the range of the window depends on the number and distribution of hydrophones (sensors), as well as on the observation time (the duration of a single frame). The main reason for employing this window is a comparatively small size of its ambiguity area, which is similar to the Gaussian function (also known as the Gabor window). The ambiguity area of this window is the smallest for all known windows. The size of the ambiguity area is particularly important because it determines the TF resolution of the obtained transforms. However, the corresponding Gabor window has to be infinite in time and in delay, and therefore it is impractical in implementation. The proposed $\mathrm{BH}$ window has slightly lower TF resolution, however it allows a real-time implementation. Other important reasons include relatively highly attenuated side-lobes of the chosen $\mathrm{BH}$ window and nonzero window derivative, as well as their analytic formulae being both known and fairly simple. There also exists the possibility to use other windows. However, as long as the incoming signals are, a priori, unknown, there is no guarantee of any benefit from their use and the proposed $\mathrm{BH}$ window is one of the best choices for the considered method.

In regard to works by Kodera's et al. [11] and in relation to (3), the channelized angular instantaneous frequency (CIF) is defined as the partial derivative of the phase $\varphi_{h}(t, \tau, \omega, \omega)$ with respect to time, in the following manner:

$$
\Omega_{h}(t, \tau, \omega, \varpi)=\varphi_{h}(t, \tau, \omega, \varpi) / \partial t
$$

Analogously, the local spatial frequency (LSF) expressed in hertz is herein defined as the partial derivative with respect to delay:

$$
\Pi_{h}(t, \tau, \omega, \varpi)=\varphi_{h}(t, \tau, \omega, \varpi) / \partial \tau
$$

where $\left|\Pi_{h}(t, \tau, \omega, \omega)\right| \leq\left|\Omega_{h}(t, \tau, \omega, \omega)\right|$, which derives from the geometrical axis of array location with respect to the direction of wave propagation. Subsequently, both the $\Omega_{h}$ and $\Pi_{h}$ distributions are used to estimate the DOA as a function of $t, \tau, \omega$, and $\omega$ following:

$$
\Phi_{h}(t, \tau, \omega, \varpi)=\arccos \left(\Pi_{h}(t, \tau, \omega, \varpi) / \Omega_{h}(t, \tau, \omega, \varpi)\right)
$$

Subsequently, the introduced reassignment, which is to be understood as energy relocation, can be performed from the frequency-spatial-frequency $(\omega-\omega)$ domain into the frequency-angle domain for each time $t$ and delay $\tau$ as follows:

$$
(t, \tau, \omega, \varpi) \rightarrow\left(t, \tau, \Omega_{h}(t, \tau, \omega, \varpi), \Phi_{h}(t, \tau, \omega, \varpi)\right) .
$$

Herein, the spectrogram's energy is defined as the squared magnitude of the transform:

$$
E_{h}(t, \tau, \omega, \varpi)=\left|U_{h}(t, \tau, \omega, \varpi)\right|^{2}=A_{h}(t, \tau, \omega, \varpi)^{2,}
$$


to a new space, where the DOA is one of the dimensions. After this reassignment, we obtain a new spectral energy distribution marked by $\varepsilon_{h}(t, \tau, \Omega, \Phi)$. Eq. (7) represents energy relocation from a raster grid to locations related to signal parameters, such as frequency and the arrival angle. Relocation vectors are obtained by processing only the STFT phase. The desired result of this process is local increase of energy concentration near the instantaneous frequency of signal components and close to the directions from which the signals arrive (bearings). The directions are represented by angles with respect to the longitudinal axis of the antenna.

\section{DIRECTIONAL CHARACTERISTIC}

In general, the arrival signals received by passive sonar can be broadband, multicomponent, noisy, and nonstationary. However, in the paper, only their DOA is within our area of interest. Therefore, we propose a consideration of the resultant four-dimensional energy distribution $\varepsilon_{h}(t, \tau, \Omega, \Phi)$ through the marginal distribution, which can be interpreted as a directional characteristic and is defined as follows:

$$
\varepsilon_{h}(t, \Phi)=\int_{\omega_{1}}^{\omega_{2}} \varepsilon_{h}\left(t, \tau_{\text {arr }} / 2, \Omega, \Phi\right) d \Omega,
$$

where $\Omega$ represents the instantaneous angular frequency of the signal, with $\omega_{1}$ and $\omega_{2}$ limiting the considered frequency band. We observe the transform in the middle of the array, where the analysing window has its maximal value, that is to say $\tau=0.5 \tau_{\text {arr }}$. Finally, the bearing estimation problem can be solved by working out the argument of energy maximum in the corresponding marginal distribution:

$$
\varnothing=\underset{\Phi}{\operatorname{argmax}} \varepsilon_{h}(t, \Phi) .
$$

\section{SEPARABILITY}

The proposed method is based upon the short-time localspatial Fourier transform. Consequently, the resolution (separability) of the method is similar to that of the transform. It depends on the number and spatial distribution of sensors, as well as the effective width of the analysing window, and especially as regards the ambiguity function. In the proposed method, a tapering window should be used as the analysing window $h(t, \tau)$. Unfortunately, this window effectively leads to array shortening and lowering in resolution within the bearing domain, compared to methods employing rectangular windows [18]. However, in the proposed method, like in other Fourier transform based methods, we can estimate the energy distributed in the joint frequency-bearing domain. Therefore, signals can be simultaneously clearly separated both in the frequency and direction of arrival. As well as this, the frequency resolution can easily be increased by extending the observation time, which is usually acceptable in passive sonar systems. This should be of assistance in separating non-coherent sources whose frequencies are even marginally different, especially for continuously functioning sonars, engines, and propellers.

\section{IMPLEMENTATION}

The formulae: (3), (4), (5), (6), and (7) introduced in the previous section have continuous representations. Their implementation on any digital machine should provide discrete equivalents. Let us assume the following notation: the values for any discrete function of selected arguments are denoted by the appropriate symbol and adjoining square brackets containing inside them the arguments. As an example, for the time-dependent two-dimensional signal let us give

$$
u[n, m]=u(t, \tau)_{t=n T_{s}, \tau=m Y_{d}}
$$

where $T_{s}=1 / r_{s}$ is the sampling interval and $Y_{d}=d_{\Delta} / v_{w}$ is the propagation delay of the acoustic wave between two neighbouring hydrophones. Both, $T_{s}$ and $Y_{d}$, are expressed in seconds. The discrete transform is denoted as follows:

$U_{h}[n, m, k, l]=U_{h}(t, \tau, \omega, \varpi)_{t=n T_{S}, \tau=m Y_{d}, \omega=k \Omega_{\Delta}, \varpi=l \Pi_{\Delta}}$

where $\Omega_{\Delta}$ and $\Pi_{\Delta}$ are the distances between lines (stems) to respective frequency and spatial frequency domains. $U_{h}[n, m, k, l]$ can be calculated by a two-dimensional FFT algorithm with zero-padding, so therefore $\Omega_{\Delta}$ and $\Pi_{\Delta}$ may be arbitrarily presumed, also being dependent, as they are, on the number of attached zeros, as well as on the number of signal samples. In the considered method, the zero-padding can be applied in order to elongate the Fourier transform up to a power of two, regardless of the window width, simultaneously increasing the number of pixels in the final imaging. However, the application of this is not necessary. The analysing window is obtained in the following manner:

$$
h[n, m]=h(t, \tau)_{t=n T_{s}, \tau=m Y_{d}}
$$

where

$$
h(t, \tau)=h_{\text {time }}(t) h_{\text {delay }}(\tau)
$$

and both $h_{\text {time }}(t)$ and $h_{\text {delay }}(\tau)$ are the Blackman-Harris windows whose widths are dependent on the observation time and array length, respectively.

There are two discrete implementations of the reassignment which are mainly known to us: interframe and intraframe, where the frame is defined as a windowed part of the signal. Their two-dimensional adaptations are introduced below. They differ particularly in their manners of phase derivative approximation, which were defined in the previous section by Eqs. (4) and (5). 


\section{INTERFRAME IMPLEMENTATION}

In the interframe approach, also referred to as the crossspectral method [7], the below solutions are used. Three adjacent (in time and delay) frames, processed by the FFT, are employed here - the middle frame being used for energy estimation based on Eq. (8), while the previous and next frame for CIF and LSF estimations, respectively:

$\check{\Omega_{h}}[n, m, k, l]=\frac{\arg U_{h}[n+1, m, k, l] \bar{U}_{h}[n-1, m, k, l]}{2 T_{s}}$

as well as:

$\check{\Pi}_{h}[n, m, k, l]=\frac{\arg U_{h}[n, m+1, k, l] \bar{U}_{h}[n, m+1, k, l]}{2 Y_{d}}$,

for $k=-K / 2, \ldots,-1,0,1, \ldots,(K-1) / 2$ and $l=-L / 2, \ldots,-1,0$, $1, \ldots,(L-1) / 2$, where $K$ and $L$ are respectively rounded upwards and downwards. $K$ and $L$ are the sizes of the discrete Fourier transforms.

\section{INTRAFRAME IMPLEMENTATION}

In the intraframe method, the phase derivatives are estimated in a different manner through the use of a differentiated window in the time domain [7]. In this method, samples from all available sensors can be used to form each frame, which is in contrast with the interframe, where at least elementary shifts along the array and in time are necessary in order to obtain derivatives.

In the intraframe method, three different analysing windows are required to obtain the Fourier transforms. The first window is a product of two Blackman-Harris windows, in a similar manner to Eq. (13). The second and third windows are obtained using the following formulae:

$$
g(t, \tau)=\frac{\partial h(t, \tau)}{\partial t}=\frac{h_{\text {time }}(t)}{\partial t} h_{\text {delay }}(\tau)
$$

and

$$
\varrho(t, \tau)=\frac{\partial h(t, \tau)}{\partial \tau}=\frac{h_{\text {delay }}(\tau)}{\partial \tau} h_{\text {time }}(t)
$$

Both $\partial h_{\text {time }}(t) / \partial t$ and $\partial h_{\text {delay }}(\tau) / \partial \tau$ can be calculated based upon the definition of the Blackman-Harris window. Finally, we obtain the transforms: $U_{h}[n, m, k, l], U_{g}[n, m, k, l]$, and $U_{\varrho}[n, m, k, l]$, which are used for both CIF and LSF estimation in the following manner:

$$
\hat{\Omega_{h}}[n, m, k, l]=\frac{2 \pi k}{T_{s} K}-\mathfrak{J} \frac{U_{g}[n, m, k, l]}{N T_{s} \bar{U}_{h}[n, m, k, l]}
$$

as well as:

$$
I \hat{T}_{h}[n, m, k, l]=\frac{2 \pi l}{Y_{d} L}-\mathfrak{J} \frac{U_{\varrho}[n, m, k, l]}{M Y_{d} \bar{U}_{h}[n, m, k, l]},
$$

for $k=-K / 2, \ldots,-1,0,1, \ldots,(K-1) / 2$ and $l=-L / 2, \ldots,-1,0,1, \ldots$, $(L-1) / 2$, where $I$ returns the imaginary part of the subsequent expression and $N$ is the number of window coefficients.

\section{COMPUTER SIMULATION}

The properties and performance of the introduced method are evaluated by the peak-to-average power ratio (PAPR) [2] and the root-mean-square error (RMSE). The simulated signals are intentionally degraded by additive white Gaussian noise (AWGN). Afterwards, bearing estimation is performed in accordance with the aforementioned instructions. The difference between the estimated and assumed DOA values is measured for various levels of the signal-to-noise ratios (SNR), as well as for different assumed directions of signal arrival. Moreover, the PAPR is calculated in order to assess the sharpness of the obtained marginal energy distributions and images.

White noise is added within the whole frequency band which is limited by half of the sampling rate $r_{s} / 2$. This process is entirely carried out by computer simulation - at least one thousand noise realizations are considered for each point of the obtained characteristics. SNR is defined as the ratio of the energy of the usable signal to total noise energy. The results are compared to conventional digital beamforming based upon the summation of accordingly delayed signals, performed within the time domain [16].

We consider an intercept sonar system with the aforementioned passive array. This system captures the following types of sounding pulses:

- pulse without frequency modulation (WFM),

- linear frequency modulated (LFM) pulse,

- hyperbolic frequency modulated (HFM) pulse.

The distance between a tracked target and the sonar is far greater than the maximal expected wavelength - the far field condition is thus satisfied. The velocity of wave propagation in the seawater is equal to $1447 \mathrm{~m} / \mathrm{s}$, which corresponds to the water temperature equal to approximately $10{ }^{\circ} \mathrm{C}[8]$. The parameters of the received signals are detailed in the appropriate sections. We take into consideration an array which consists of sixteen hydrophones set apart from each other by nine and half centimetres, a distance connected to the frequency of the considered signals. The assumed sampling rate is fifteen kilo samples per second. Graphical results are also presented as visual comparison of the proposed method and conventional beamforming.

In the performed experiment, the introduced method is defined by the following procedure:

1. STFT calculation.

2. Energy, CIF, LSF, and DOA estimation based on STFT.

3. Composition of reassignment vectors using the DOA and CIF as orthogonal components.

4. Energy relocation according to reassignment vectors.

5. Estimation of the new energy density in the frequencyangle domain. 
6. Preparation of marginal distribution of this energy in the bearing domain.

7. The argument of the global maximum of the marginal distribution being the final result.

In all variants, the global maximum can be changed to local maxima if additional signals from different directions are anticipated.

\section{CONVENTIONAL BEAMFORMING IN TIME DOMAIN}

The proposed method is compared with conventional beamforming performed in the time domain by the sum values of accordingly delayed discrete signals. The signals derived from each hydrophone are interpolated by a four-point digital filter in order to obtain fractional delay corresponding to the appropriate beam and direction [16]. Finally, we specify bearing by looking for the argument of the maximum of the directional characteristic. To this end, we use the golden section search, where the tolerance parameter is assumed equal to one hundredth degree. The resultant characteristic is averaged in such a way that the analysis covers the same parts of the signal in the two compared methods.

\section{ANALYSIS OF WFM PULSES}

A pulse without frequency modulation, also known as a mono-tone burst or finite duration impulse, is one of the simplest sounding excitations used in the sonar technique. In the simulation presented in Figure 3, we use a pulse without frequency modulation (WFM) whose constant frequency is equal to five kilohertz and duration equal to ten milliseconds. Furthermore, the Tukey window is applied in order to model the non-zero duration of the rising and falling edges. This window is applied for shaping all the considered pulses in the presented experiments.

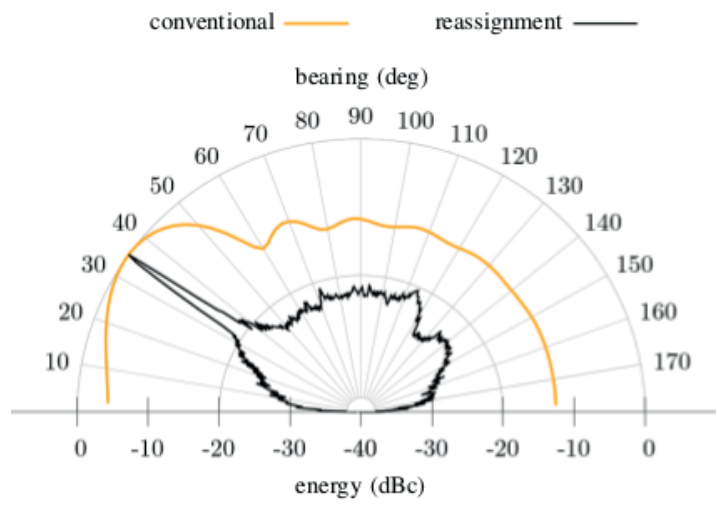

Fig. 3. Comparison of marginal energy distribution after reassignment carried out by the interframe variant (black) with the directional characteristic obtained by conventional beamforming performed in the time domain (orange). A single tone burst in the presence of AWGN is analysed. SNR is equal to approximately 0 decibels. True direction of arrival is assumed equal to 35 degrees.

\section{ANALYSIS OF LFM PULSES}

In general, the frequency modulation allows us to efficiently use the pulse compression carried out by matched filtering. However, in the considered simulation we assume that the parameters of the matched filter are unknown. Therefore, optimal pulse compression is not applied at either this, or any subsequent point. We assess the capabilities of the introduced method for LFM signal analysis. The signal parameters which were employed in this experiment are the following:

- duration - $10 \mathrm{~ms}$,

- mean frequency - $5 \mathrm{kHz}$,

- frequency bandwidth $-5 \mathrm{kHz}$,

- frequency band $-2.5 \div 7.5 \mathrm{kHz}$,

The results of this analysis are shown in Figure 4.
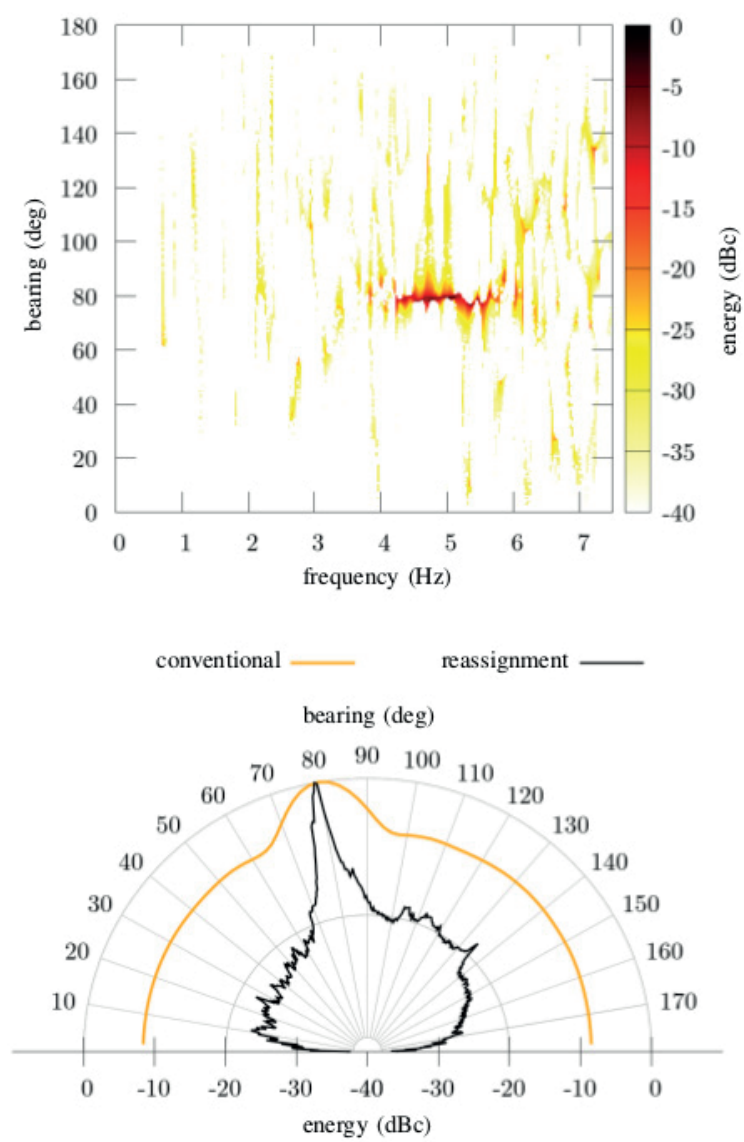

Fig. 4. Reassigned spectrogram estimated by the intraframe variant (top), and the corresponding comparison of marginal energy distributions after reassignment (black; bottom) with the directional characteristic obtained by conventional beamforming performed in the time domain (orange; bottom). A single LFM pulse in the presence of AWGN is herein analysed. SNR is equal to approximately 0 decibels. True direction of arrival is equal to 80 degrees.

\section{ANALYSIS OF HFM PULSES}

Hyperbolic frequency modulation is also known as logarithmic phase modulation and linear period modulation. This technique is widely used in wideband active sonars designed for minimizing the degradation caused by Doppler shifting of the return signal, in the instance when the 
source and target are in relative motion [17]. In the present simulation, we assumed the same parameters as for the LFM signals considered in Section 5.3. The results of the analysis of this signal are presented in Figure 5.

\section{MULTICOMPONENT SIGNALS}

Signal separability is especially important when a number of unknown targets on similar directions have to be detected and specified. In general, conventional beamforming has superior separability in the bearing domain, because a rectangular window is used in this method. However, in some situations the here introduced method can give better results, compared to conventional beamforming, on the condition that no additional selective filtering is applied. This is possible because we base upon separation in the joint frequency-bearing domain. This situation is presented in Figure 6, in which the constant frequency pulse is added as the interfering signal to the LFM pulse. The signals under consideration slightly overlap in the frequency domain. However, both signals are clearly separated by the proposed method and both directions of arrival can be estimated, which is in contrast to the conventional method. The LFM pulse has the same parameters as in Section 5.3. The frequency of the WFM pulse is equal to three and half kilohertz.
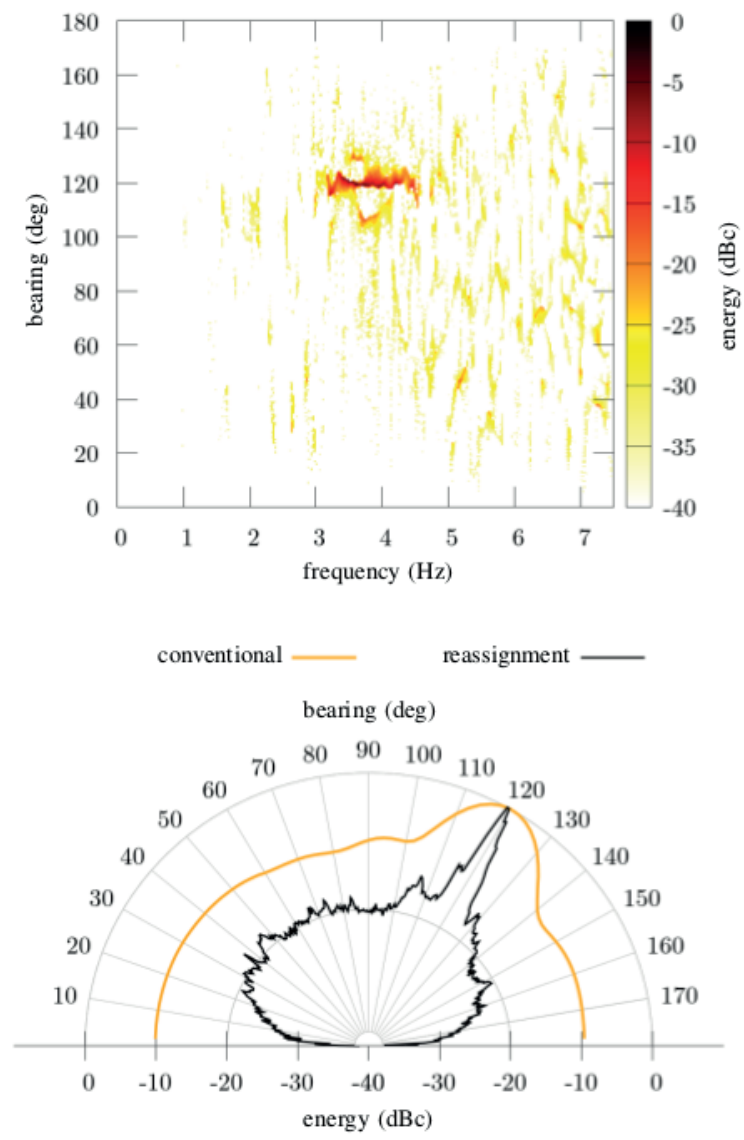

Fig. 5. Reassigned spectrogram estimated by the interframe variant (top), and the corresponding comparison of marginal energy distributions after reassignment (black; bottom) with the directional characteristic obtained by conventional beamforming performed in the time domain (orange; bottom). A single HFM pulse in the presence of AWGN is herein analysed. SNR is equal to approximately 0 decibels. True direction of arrival is equal to 120 degrees.

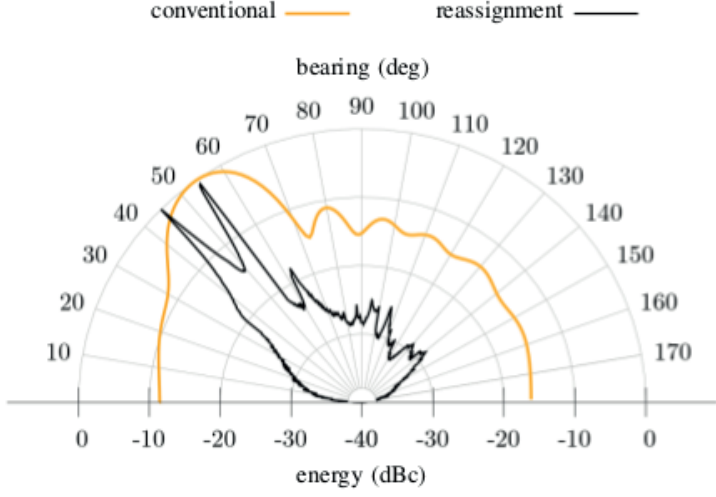

Figure 6. Comparison of marginal energy distributions after reassignment (black) with the directional characteristic obtained by conventional beamforming performed in the time domain (orange). LFM and WFM pulses in the presence of AWGN are herein analysed. SNR is equal to approximately six decibels. True directions of signal arrival are equal to 45 and 55 degrees, respectively.

\section{CREST FACTOR}

The peak-to-average power ratio is also referred to as the crest factor, or peak factor. In the considered context, the crest factor is used to assess the sharpness of the obtained distributions and imaging [2]. A high level of this factor is interpreted as constituting either high contrast in the usable signal representation in final imaging, or a clear peak in marginal distribution in the bearing domain. In this experiment, the results of which are shown in Figure 7, as well as in Tables 1 and 2, all three kinds of pulses (WFM, LFM, and HFM) were considered for different directions of arrival, window widths, and SNR levels. In addition, the crest factor can be utilized to find the optimal width of the analysing window in the time domain. The optimal absolute window width is equal to approximately five thousandths of a second for the LFM and HFM pulses, and about two times greater for the WFM pulse. This value corresponds to the duration of the analysed pulses. In general, the optimal window width may vary for different signals and varying parameters. The results in Tables 1 and 2 were obtained for the analysing window whose width is equal to five thousandths of a second, which is close to the optimal value for LFM and HFM pulses. In such a situation, signal parameters are the same as those reported in Sections 5.3 and 5.4. In all rows of these tables, the achieved crest factors are higher for the proposed method based upon reassignment than for conventional beamforming.

Table 1. Crest factor obtained for reassignment and conventional beamforming for selected DOA and for $S N R \approx-6$ decibels

\begin{tabular}{|c|c|c|c|}
\hline & & \multicolumn{2}{|c|}{ PAPR } \\
\hline pulse type & DOA & conventional & reassignment \\
\hline & degrees & $\mathrm{dB}$ & $\mathrm{dB}$ \\
\hline WFM & 45 & 2.362 & 14.076 \\
\hline LFM & 45 & 3.319 & 12.828 \\
\hline HFM & 45 & 4.317 & 12.094 \\
\hline WFM & 75 & 2.449 & 14.525 \\
\hline LFM & 75 & 3.518 & 13.471 \\
\hline HFM & 75 & 4.655 & 12.858 \\
\hline
\end{tabular}




\begin{tabular}{|c|c|c|c|}
\hline & & \multicolumn{2}{|c|}{ PAPR } \\
\hline pulse type & DOA & conventional & reassignment \\
\hline & degrees & $\mathrm{dB}$ & $\mathrm{dB}$ \\
\hline WFM & 90 & 2.502 & 14.669 \\
\hline LFM & 90 & 3.579 & 13.923 \\
\hline HFM & 90 & 4.699 & 13.112 \\
\hline
\end{tabular}

Tab 2. Crest factor obtained for reassignment and conventional method for $D O A=75$ degrees and for selected SNR levels

\begin{tabular}{|c|c|c|c|}
\hline & & \multicolumn{2}{|c|}{ PAPR } \\
\hline pulse type & SNR & conventional & reassignment \\
\hline & $\mathrm{dB}$ & $\mathrm{dB}$ & $\mathrm{dB}$ \\
\hline WFM & -5.3 & 2.667 & 14.728 \\
\hline WFM & 5.8 & 8.738 & 21.977 \\
\hline LFM & -5.3 & 3.503 & 13.145 \\
\hline LFM & 5.8 & 8.953 & 20.626 \\
\hline HFM & -5.3 & 4.991 & 12.759 \\
\hline HFM & 5.8 & 9.28 & 20.227 \\
\hline
\end{tabular}

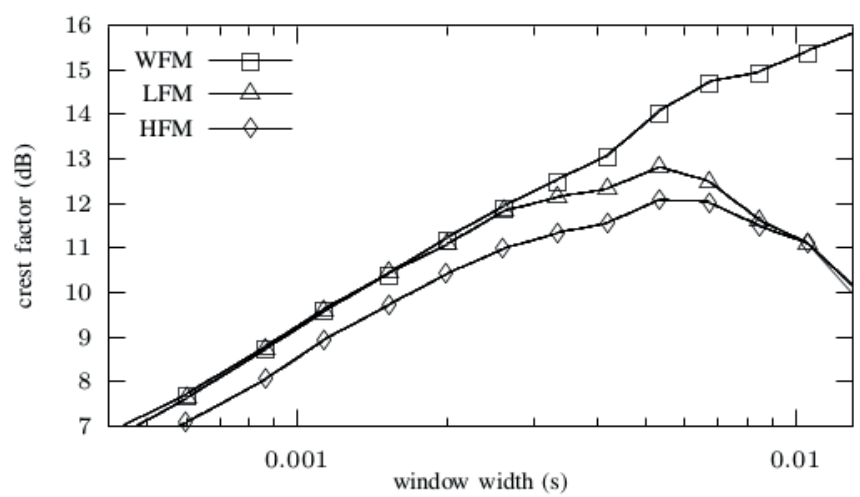

Fig. 7. Comparison of crest factor as a function of absolute window width for WFM, LFM and HFM pulses. Crest factor is calculated for the marginal energy distribution in the presence of noise. SNR is equal to approximately minus six decibels. True direction of arrival is assumed equal to forty-five degrees.

\section{ROOT MEAN SQUARE ERROR}

The root mean square error is estimated in order to evaluate the accuracy of the introduced method. The RMSE curves as functions of the signal-to-noise ratio are presented in Figure 8. The simulations were performed for three directions $(45,75$, and 90 degrees) and three considered pulses (WFM, LFM, and HFM). The used window had the optimal width being equal to approximately five thousandths of a second, which was estimated in the previous section. In all considered situations, the introduced method has significantly worse accuracy than conventional beamforming, which is its serious drawback. Table 3 collates errors for inter- and intraframe implementations. The DOA is assumed equal to seventy five degrees, and SNR is equal to approximately minus half decibels. The results indicate that the intraframe variant is slightly better.
Tab. 3. Root mean square errors obtained for interframe and intraframe for selected simulation parameters

\begin{tabular}{|c|c|c|c|c|}
\hline & & & \multicolumn{2}{|c|}{ RMSE } \\
\hline pulse type & DOA & SNR & interframe & intraframe \\
\hline & degrees & $\mathrm{dB}$ & degrees & degrees \\
\hline WFM & 75 & -0.5 & 0.717 & 0.677 \\
\hline LFM & 75 & -0.5 & 1.017 & 0.742 \\
\hline HFM & 75 & -0.5 & 1.175 & 0.973 \\
\hline
\end{tabular}

\section{DISCUSSION}

The performed experiments demonstrate high sensitivity of the introduced method to the influence of noise. The conventional beamformer is significantly superior in this regard. There are two main reasons for this. Namely, the use of the analysing tapering window causes a decrease in the effective length of the array and reduces the final resolution. Therefore, a uniformly distributed AWGN has a greater impact. Unfortunately, it is not recommended to omit the use of the window. Secondly, both CIF and LSF, as well as the STFT energy of different signals derived from different noncoherent sources, in accordance with the Heisenberg-Gabor limit, cannot be completely independent in the considered domain. This is a well-known problem as regards the timefrequency analysis, which is related to the ambiguity function and the choice of the analysing window.
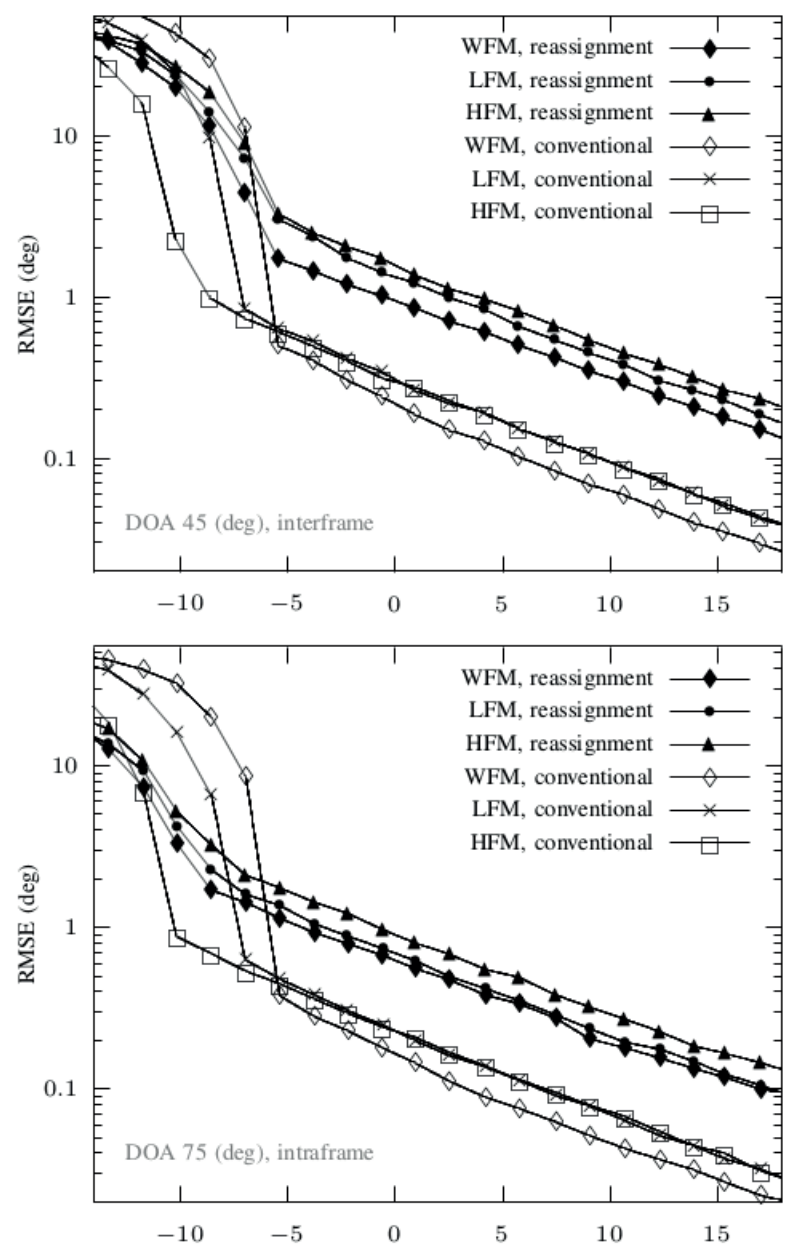


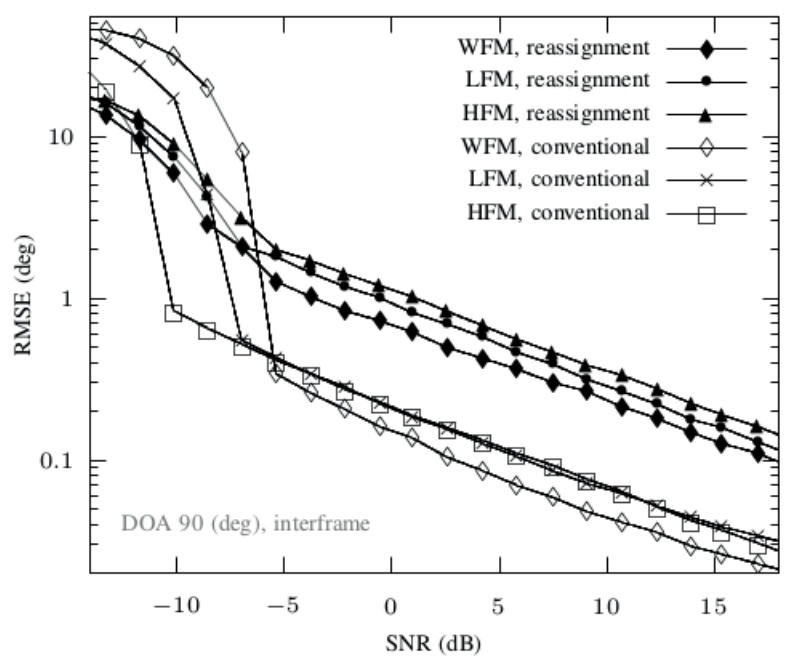

Fig. 8: Comparison of the root mean square error as a function of SNR for both conventional and reassignment methods in the presence of AWGN. True directions of arrival are assumed equal to 45, 75, and 90 degrees, respectively. The interframe and intraframe variants are tested.

On the other hand, in some situations (see for example Fig. 6), the introduced method yields superior results in the separation of two (or more) signals whose frequency bands partially overlap. This is achieved by simultaneous analysis in the joint frequency-bearing domain, while a conventional beamformer only provides separation in the bearing domain. Similar results are also possible for the conventional solution through additional band filtering performed in the time domain. However, this involves complication of the algorithm.

The presented method can be useful especially in passive sonar monitoring to estimate signal parameters, which can be subsequently used for further analysis or processing. Signals which can be analysed in this manner include also noise generated by ships. Comparing the conditions reported in [23], [24] to the results presented in Figure 8 gives us a considerable chance that the method can also be applied online for monitoring coastal waters of the Baltic Sea [25]. This is all the more so that we can find the information in scientific literature about the use of a linear array in the shallow water [20]. We are going to continue our research in this area.

\section{CONCLUSION}

The paper demonstrates the possibility of using twodimensional (double frequency) reassignment for bearing estimation. The reassignment is based upon the Fourier transform and is carried out in the joint frequency-bearing domain, which enables to obtain highly concentrated energy distributions, as well as clear and sharp imaging. We have used the crest factor in order to assess this concentration. The results were compared to conventional beamforming performed in the time domain. In all the experiments, the crest factor is significantly higher than that for the conventional method. This confirms that the resultant images generated by the introduced method are sharper and more clear. The crest factor was also used to find the optimal width of the analysing window, which is related to parameters of pulses under consideration. In sonar and radar techniques, a high crest factor after pulse compression is usually required to ensure good quality detection [2], [13].

Two implementations of reassignment were examined, which were the interframe and the intraframe. They originate from the time-frequency reassignment and provide very similar results, however the intraframe variant turned out slightly better, as a result of the fact that all sensors were used in calculations of every single Fourier transform. This is in contrast to the interframe, which utilizes at least one less sensor, since elementary shift along the array is necessary in order to estimate the phase derivative. As a result of this, the effective length of the array is fractionally shorter approximately six percent for sixteen hydrophones.

The introduced method has been regarded as being both fast and efficient, due to the fact that its implementation is based upon a two-dimensional FFT algorithm [22]. The analysis in the frequency and bearing domains was conducted in a single-phase, which may also increase the computing performance in comparison with two-stage processing which includes conventional time and spatial filtering.

\section{ACKNOWLEDGMENT}

The authors would like to thank Dr. Mirosław Rojewski and Prof. Roman Salamon for their suggestions and discussion on this topic.

\section{REFERENCES}

1. F. Auger, P. Flandrin, Yu-Ting Lin, S. McLaughlin, S. Meignen, T. Oberlin, Hau-Tieng $\mathrm{Wu}$, "Time-frequency reassignment and synchrosqueezing: an overview", IEEE Signal Proc. Mag., vol. 30, no. 6, pp. 32-41, 2013.

2. J.S. Byrnes, "Quadrature Mirror Filters, Low Crest Factor Arrays, Functions Achieving Optimal Uncertainty Principle Bounds, and Complete Orthonormal Sequences - A Unified Approach", Applied and Computational Harmonic Analysis, vol. 1, no. 3, pp. 261-266, 1994.

3. Z. Chen, J. Sun, H. Hou, "Phase difference method for DOA estimation", J. of Marine Science and Application, vol. 9, no.4, pp.445-450, 2010.

4. C. Chi, Z. Li, Q. Li, "Fast broadband beamforming using nonuniform Fast Fourier Transform for underwater realtime 3-d acoustical imaging", IEEE J. Ocean. Eng., vol.41, no.2, pp.249-246, 2016.

5. K. Czarnecki, "The instantaneous frequency rate spectrogram”, Mech. Syst. Signal Process., vol. 66-67, pp.361-373, 2016. 
6. K. Czarnecki, W. Leśniak, “The synchrosqueezing method in the bearing estimation of stationary signals for a passive sonar system with a towed array", Hydroacoustics, vol. 18, pp. 41-46, 2015

7. S.A. Fulop, K. Fitz, "Algorithms for computing the time-corrected instantaneous frequency (reassigned) spectrogram, with applications", J. Acoust. Soc. Amer. (JASA), vol. 119, no. 1, pp.360-371, 2006.

8. V.A. Del Grosso, "Tables of the speed of sound in open ocean water (with Mediterranean Sea and Red Sea applicability)", J. Acoust. Soc. Amer. (JASA), vol.53, no.5, pp.1384-1401, 1973.

9. S. Haykin, Radar array processing for angle of arrival estimation in Array Signal Processing, pp. 149-292, Prentice-Hall, 1985.

10. L.B. Jackson, H. Chien, "Frequency and bearing estimation by two-dimensional linear prediction”, proc. IEEE International Conference on Acoustics, Speech, and Signal Processing, vol. 4, pp. 665-668, 1979.

11. K. Kodera, C.D. Villedary, R. Gendrin, "A new method for the numerical analysis of non-stationary signals", Phys. Earth Planet. In., vol.12, pp.142-150,1976.

12. H. Krim, M. Viberg, "Two decades of array signal processing research”, IEEE Signal Proc. Mag., vol. 13, no. 4, pp.67-94, 1996.

13. A. De Maio, Y. Huang, M. Piezzo, S. Zhang, A. Farina, "Design of Optimized Radar Codes with a Peak to Average Power Ratio Constraint”, IEEE Tran. Signal Process., vol. 59, no. 6, pp. 2683-2697, 2011.

14. J. Marszal, Digital signal processing applied to the modernization of Polish Navy sonars, Polish Maritime Research, vol. 21, no. 2, pp. 65-75, 2014.

15. B. Maranda, "Efficient digital beamforming in the frequency domain”, J. Acoust. Soc. Amer., vol. 86, no. 5, pp. 1813-1819, 1989.

16. R.G. Pridham, R.A. Mucci, "Digital interpolation beamforming for low-pass and bandpass signals", in Proceedings of the IEEE, vol. 67, no. 6, pp. 904-919, 1979.

17. M. Readhead, Calculations of the sound scattering of hyperbolic frequency modulated chirped pulses from fluidfilled spherical shell sonar targets, technical note, Maritime Operations Division, Defence Science and Technology Organisation, Australia, 2010.
18. P. Rudnick, "Digital Beamforming in the Frequency Domain”, J. Acoust. Soc. Amer., vol. 46, no. 5, pp. 1089$1090,1969$.

19. H. Slabbekoorn, N. Bouton, I. Van Opzeeland, A. Coers, C. ten Cate, A.N. Popper, "A noisy spring: the impact of globally rising underwater sound levels on fish", Trends in Ecology \& Evolution, vol. 25, no. 7, pp. 419-427, 2010.

20. W. Szymczak, E. Kozaczka, G. Grelowska, I. Gloza, and S. Kozaczka, "The shallow sea experiment with usage of linear hydrophone array", The Journal of the Acoustical Society of America, vol. 133, no. 5, pp. 3439-3439, 2013.

21. E. Tuncer, B. Friedlander, Classical and Modern Direction of Arrival Estimation, Elsevier, Burlington, MA, 2009

22. J.R. Williams, "Fast beam-forming algorithm", J. Acoust. Soc. Amer. (JASA), vol. 44, no. 5, pp. 1454-1455, 1968.

23. G. Grelowska, E. Kozaczka, S. Kozaczka, W. Szymczak, "Underwater Noise Generated by a Small Ship in the Shallow Sea", Archives of Acoustics, vol. 39, no. 3, pp. 351356, 2013.

24. G. Grelowska, "Study of seasonal acoustic properties of sea water in selected waters of the southern Baltic", Polish Maritime Research, vol. 23, no. 1, pp. 25-30, 2016.

25. G. Grelowska, E. Kozaczka, S. Kozaczka, W. Szymczak, "Gdansk Bay sea bed sounding and classification of its results”, Polish Maritime Research, vol. 20, no. 3, pp. 45-50, 2013.

\section{CONTACT WITH THE AUTHORS}

\section{Krzysztof Czarnecki}

e-mail:krzycz@eti.pg.gda.pl

Gdansk University of Technology, Narutowicza 11/12, 80-233 Gdańsk

POLAND

Wojciech Lesniak

e-mail:wojlesni1@pg.edu.pl

Gdansk University of Technology, Narutowicza 11/12, 80-233 Gdańsk

POLAND 